\title{
A Novel Algorithm for Fitting 3-D Active Appearance Models: Applications to Cardiac MRI Segmentation
}

\author{
Alexander Andreopoulos and John K. Tsotsos \\ York University, Dept. of Computer Science and Engineering, \\ Centre for Vision Research, Toronto Ontario, M3J 1P3, Canada \\ \{alekos, tsotsos\}@cs.yorku.ca
}

\begin{abstract}
We present an efficient algorithm for fitting three dimensional (3-D) Active Appearance Models (AAMs). We do so, by introducing a 3-D extension of a recently proposed method that is based on the inverse compositional image alignment algorithm. We demonstrate its applicability for the segmentation of the left ventricle in short axis cardiac MRI. We perform experiments to evaluate the speed and segmentation accuracy of our algorithm on a total of 1473 cardiac MR images acquired from 11 patients. The fitting is around 60 times faster than standard Gauss-Newton optimization, with a segmentation accuracy that is as good as, and often better than Gauss-Newton.
\end{abstract}

\section{Introduction}

Active Appearance Models (AAMs) provide a promising method for the interpretation of medical images [3], 4]. There is interest in the use of 3-D Active Appearance Models for the segmentation of the left ventricle from short axis cardiac MRI [5], due to AAMs' ability to learn the 3-D structure of the heart and not lead to unlikely segmentations [7].

The algorithms described in the literature for fitting AAMs, are either robust but inefficient gradient descent type algorithms, or efficient but ad-hoc algorithms. Fitting AAMs using standard optimization methods is inefficient, due to the high number of parameters needing to be optimized. This problem is exacerbated with 3-D AAMs since such models can use 50-100 parameters. To deal with this, efficient algorithms for fitting AAMs have been developed [4. Under such formulations, we look for a constant matrix $\mathbf{R}$ such that if the current fitting error between the AAM and the image is $\delta \mathbf{t}$, the update to the AAM parameters is $\delta \mathbf{p}=\mathbf{R} \delta \mathbf{t}$ However, the fitting accuracy and convergence rates of such algorithms are often unsatisfactory. Such methods lack a sound theoretical basis since in general there is no reason why the error measure $\delta \mathbf{t}$ should uniquely identify the update $\delta \mathbf{p}$ of the parameters.

Recently, a novel algorithm for fitting 2-D AAMs was introduced in [6]. Its applicability was demonstrated on artificial data and for face tracking. However, as it is cited in [6], there was no known way of extending the algorithm to 
higher dimensions since a certain argument used in the paper applied only to 2-D similarity transformations. In this paper we present an extension of the algorithm for the fitting of 3-D AAMs on short axis cardiac MRI. By definition, short axis cardiac MR images are such that the long axis of the heart is perpendicular to the acquisition image plane. This means that during the AAM fitting we need to rotate our model only around the long axis of the heart. We take advantage of this fact to design an efficient fitting algorithm.

We perform experiments comparing our algorithm with Gauss-Newton based optimization, which is generally known as one of the most accurate and reliable optimization algorithms for such problems [1]. We observe a 60 fold improvement in the fitting speed, with a segmentation accuracy that is as good - and in many cases better - as brute force Gauss-Newton optimization. Our algorithm's border positioning errors are significantly smaller than the errors reported for other $3-\mathrm{D}$ AAMs [7] which use the constant matrix approach for the fitting.

\section{3-D AAMs}

Figure 1(a) shows a short axis cardiac MR image. A stack of such images gives a volumetric representation of the heart. Manual segmentations of the left ventricle provide us contours representing the endocardium and epicardium of the left ventricle. By uniformly sampling each of these contours at $i_{0}$ points along their arclength, starting from the closest point to the posterior junction of the left and right ventricles, each contour is represented by a set of landmarks. By stacking the landmarks on top of each other we obtain a 3-D representation of the left ventricle's endocardium and epicardium, as shown in Figure 1(b). However, the number of images intersecting the left ventricle is not the same across every patient. Therefore, we need to interpolate between the contours so that every 3 -D model is made up of the same number of slices. If we want to create a slice at height $z_{0}$ located between two slices, we can simply do the following: From the line segment joining the $i^{t h}$ landmark in the two slices, we find the location with height $z_{0}$. This gives us the $i^{\text {th }}$ landmark in the new slice. In our implementation, we created 15 contour slices, evenly sampled along the z-axis, located between the apex and basal contours.

If we have a set of $\mathrm{N}$ sample shapes, and each sample consists of $m$ landmarks, we can represent each shape sample as a $3 m$ dimensional vector. By applying principal component analysis (PCA) on the distribution of the shape vectors, any shape $\mathbf{s}$ out of the $\mathrm{N}$ shapes can be approximated as

$$
\mathbf{s} \approx \mathbf{s}_{0}+\sum_{i=1}^{n} p_{i} \mathbf{s}_{i}
$$

for some $\mathbf{p}=\left(p_{1}, \ldots, p_{n}\right) \in \Re^{n}$, where $\mathbf{s}_{\mathbf{0}}$ is the mean shape vector (a.k.a base mesh), and $\mathbf{s}_{i}$ indicates the $i^{\text {th }}$ eigenvector. We are summing over $n$ eigenvectors $\mathbf{s}_{i}$ such that they explain around $90 \%-95 \%$ of the shape variation. 
When building a 2-D AAM, we need to make sure that the shapes are aligned with each other so that we remove any difference between two shapes due to a similarity transform. See [3] for more details. This leads to more compact AAMs, which can be described by a smaller number of parameters. In [6] and in our algorithm, this is a necessary step since, by removing the similarity transform from the training set shapes, the $\mathbf{s}_{i}$ vectors will be orthogonal to a subspace of the 3-D similarity transforms of $\mathbf{s}_{0}$. In our algorithm, this was accomplished by using an iterative alignment procedure as described in [3], only that in this case we aligned the shapes with respect to translation, scaling and rotation around only the z-axis. We did not align the shapes with respect to $\mathrm{x}$ and $\mathrm{y}$ axis rotations since we only wanted our model to handle rotations around the z-axis.

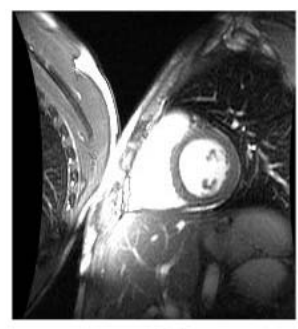

(a)

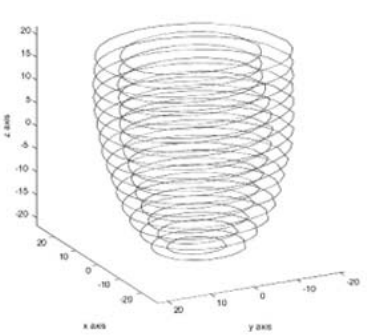

(b)

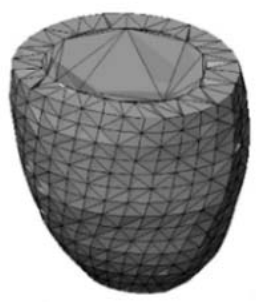

(c)

Fig. 1. (a)Short axis cardiac MRI. (b)Endocardial and epicardial landmarks stacked on each other. Shown as curves for greater clarity. The mean of these landmarks is given by vector $\mathbf{s}_{0}$. (c)Tetrahedrization of $\mathbf{s}_{0}$. Each tetrahedron represents a part of the myocardial muscle or a part of the left ventricle's blood pool

We need to model the appearance variation of the shape. We first manually tetrahedrize $\mathbf{s}_{0}$, as shown in Figure 1(c). This splits the left ventricular volume enclosed by $\mathbf{s}_{0}$ into tetrahedra. The same landmark connectivity defining the tetrahedra of $\mathbf{s}_{0}$ can be used to define the tetrahedrization of any shape variation resulting from Eq. (1). Then, we use these tetrahedra to sample the appearance enclosed by each training shape [7. Let the mean appearance we get by averaging the sampled appearances be $A_{0}(\mathbf{x})$ and the $k$ eigenvectors we found by PCA, describing around $90 \%-95 \%$ of the appearance variation, be $A_{1}(\mathbf{x}), A_{2}(\mathbf{x}), \ldots, A_{k}(\mathbf{x})$ (where $\mathbf{x}$ denotes the appearance coordinate in the base model $\mathbf{s}_{0}$ coordinate system). For different $b_{i}$ values

$$
A(\mathbf{x})=A_{0}(\mathbf{x})+\sum_{i=1}^{k} b_{i} A_{i}(\mathbf{x})
$$

defines the appearance variations the model has learned from our training data. 


\section{The Inverse Compositional Algorithm}

There is a wealth of literature on image alignment algorithms and the reader is referred to [1,2] and the references therein for an overview. The inverse compositional approach has been shown to provide fast and reliable image alignment.

Assume we have a template $A_{0}(\mathbf{x})$ that we want to align with an image $I(\mathbf{x})$. In the compositional framework for image alignment we compute a warp $\mathbf{W}(\mathbf{x} ; \boldsymbol{\Delta} \mathbf{p})$ that is composed with the current warp $\mathbf{W}(\mathbf{x} ; \mathbf{p})$ (where $\mathbf{p}$ are warp parameters and $\mathbf{x}$ denotes pixel/voxel coordinates), in order to find the update parameters $\Delta \mathbf{p}$ minimizing

$$
\sum_{\mathbf{x} \in \operatorname{Domain}\left(A_{0}\right)}\left[I(\mathbf{W}(\mathbf{W}(\mathbf{x} ; \boldsymbol{\Delta} \mathbf{p}) ; \mathbf{p}))-A_{0}(\mathbf{x})\right]^{2} .
$$

In the inverse compositional approach we are trying to minimize

$$
\sum_{\mathbf{x} \in \operatorname{Domain}\left(A_{0}(W)\right)}\left[I(\mathbf{W}(\mathbf{x} ; \mathbf{p}))-A_{0}(\mathbf{W}(\mathbf{x} ; \boldsymbol{\Delta} \mathbf{p}))\right]^{2} .
$$

The solution to this least squares problem is approximately [1]:

$$
\boldsymbol{\Delta} \mathbf{p}=\mathbf{H}^{-1} \sum_{\mathbf{x} \in \operatorname{Domain}\left(A_{0}\right)}\left[\nabla A_{0} \frac{\partial \mathbf{W}}{\partial \mathbf{p}}\right]^{T}\left[I(\mathbf{W}(\mathbf{x} ; \mathbf{p}))-A_{0}(\mathbf{x})\right]
$$

where

$$
\mathbf{H}=\sum_{\mathbf{x} \in \operatorname{Domain}\left(A_{0}\right)}\left[\nabla A_{0} \frac{\partial \mathbf{W}}{\partial \mathbf{p}}\right]^{T}\left[\nabla A_{0} \frac{\partial \mathbf{W}}{\partial \mathbf{p}}\right]
$$

and $\frac{\partial \mathbf{W}}{\partial \mathbf{p}}$ is evaluated at $(\mathbf{x} ; \mathbf{0})$. It can be shown that within first order, under the condition of a fairly "smooth" warping function $\mathbf{W}$, (4) is equal to

$$
\sum_{\mathbf{x} \in \operatorname{Domain}\left(A_{0}\right)}\left[I\left(\mathbf{W}\left(\mathbf{W}^{-\mathbf{1}}(\mathbf{x} ; \boldsymbol{\Delta} \mathbf{p}) ; \mathbf{p}\right)\right)-A_{0}(\mathbf{x})\right]^{2} .
$$

This means that the $\boldsymbol{\Delta} \mathbf{p}$ in (5) can also be used to minimize (7). Notice that (7) is an image alignment error measure of the form (3). So once we have found $\Delta \mathbf{p}$ we can update the warp by

$$
\mathbf{W}(\mathbf{x} ; \mathbf{p}) \leftarrow \mathbf{W}(\mathbf{x} ; \mathbf{p}) \circ \mathbf{W}^{-1}(\mathbf{x} ; \Delta \mathbf{p})
$$

and go to Eq. (5) to perform another iteration of the image alignment algorithm. This is a very efficient algorithm, known as the inverse compositional algorithm [2]. It is efficient because we can precompute $\nabla A_{0} \frac{\partial \mathbf{W}}{\partial \mathbf{p}}$ and $\mathbf{H}$. This algorithm can easily be used for fitting templates $A(\mathbf{x})$ as described in section 2 . In that case, if in Eq. (4) above, $A_{0}(\mathbf{x})$ is replaced by $A(\mathbf{x})$, with appearance variation as described in section 2 , then within first order the error is equal to [2]:

$$
\sum_{\mathbf{x} \in \operatorname{Domain}\left(A_{0}\right)}\left[\operatorname{proj}_{\operatorname{span}\left(A_{i}\right)^{\perp}}\left(I\left(\mathbf{W}\left(\mathbf{W}^{-\mathbf{1}}(\mathbf{x} ; \boldsymbol{\Delta} \mathbf{p}) ; \mathbf{p}\right)\right)-A_{0}(\mathbf{x})\right)\right]^{2}
$$


where $\operatorname{span}\left(A_{i}\right)^{\perp}$ denotes the space orthogonal to $A_{1}, \ldots, A_{k}$. We minimize expression (9) by using expressions (5), (6), only that now we project each column of $\nabla A_{0} \frac{\partial \mathbf{W}}{\partial \mathbf{p}}$ onto $\operatorname{span}\left(A_{i}\right)^{\perp}$ and use this projected matrix instead of $\nabla A_{0} \frac{\partial \mathbf{W}}{\partial \mathbf{p}}$.

\section{Inverse Compositional Fitting of 3-D AAMs}

In this section we show how to fit 3-D AAMs using the inverse compositional approach. In section 4.1 we show how to extend [6] to fit 3-D AAMs with no global similarity transformations. In section 4.2 we show how to fit a 3 -D AAM when we allow translations, rotations around only one axis - by convention we will be rotating around the $\mathrm{z}$-axis - and scaling of the coordinate axes.

\subsection{Fitting Without Global Shape Transform}

We now show how the inverse compositional algorithm can be used for fitting 3-D AAMs without taking care of any global shape similarity transformations (translation, scalings and rotations).

We first need to define $\mathbf{W}(\mathbf{x} ; \mathbf{p})$, where $\mathbf{p}$ denotes the current landmarks model parameters from Eq. (1), and the $\mathbf{x}=(x, y, z)^{T}$ parameter denotes a point in the base mesh $\mathbf{s}_{\mathbf{0}}$. Then $\mathbf{W}(\mathbf{x} ; \mathbf{p})$ denotes the warping of $\mathbf{x}$ under the current warp parameters $\mathbf{p}$. As mentioned above, every base mesh voxel $\mathbf{x}$ lies in a tetrahedron $\mathbf{T}_{\mathbf{0}}$ defined by the vertices $\left(x_{i}^{0}, y_{i}^{0}, z_{i}^{0}\right),\left(x_{j}^{0}, y_{j}^{0}, z_{j}^{0}\right),\left(x_{k}^{0}, y_{k}^{0}, z_{k}^{0}\right)$, $\left(x_{l}^{0}, y_{l}^{0}, z_{l}^{0}\right)$. If the current shape parameters of the model are $\mathbf{p}$, then let the vertices of the deformed tetrahedron $\mathbf{T}_{\mathbf{1}}$ be $\left(x_{i}, y_{i}, z_{i}\right),\left(x_{j}, y_{j}, z_{j}\right),\left(x_{k}, y_{k}, z_{k}\right)$, $\left(x_{l}, y_{l}, z_{l}\right)$ which were computed from Eq. (1). W(x; p) computes the affine transformation of $\mathbf{x}$ from $\mathbf{T}_{\mathbf{0}}$ to $\mathbf{T}_{\mathbf{1}}$. If $\alpha_{i}, \alpha_{j}, \alpha_{k}, \alpha_{l}$ denote the barycentric coordinates of $\mathbf{x}$ in $\mathbf{T}_{\mathbf{0}}$ given by

$$
\left(\begin{array}{c}
\alpha_{i} \\
\alpha_{j} \\
\alpha_{k} \\
\alpha_{l}
\end{array}\right)=\left(\begin{array}{cccc}
x_{i}^{0} & x_{j}^{0} & x_{k}^{0} & x_{l}^{0} \\
y_{i}^{0} & y_{j}^{0} & y_{k}^{0} & y_{l}^{0} \\
z_{i}^{0} & z_{j}^{0} & z_{k}^{0} & z_{l}^{0} \\
1 & 1 & 1 & 1
\end{array}\right)^{-1}\left(\begin{array}{c}
x \\
y \\
z \\
1
\end{array}\right)
$$

(by the definition of barycentric coordinates $\alpha_{i}+\alpha_{j}+\alpha_{k}+\alpha_{l}=1$ and $0 \leq$ $\left.\alpha_{i}, \alpha_{j}, \alpha_{k}, \alpha_{l} \leq 1\right)$, the affine transformation of $\mathbf{x}$ is $\mathbf{W}(\mathbf{x} ; \mathbf{p})$ and is given by:

$$
\alpha_{i}\left(x_{i}, y_{i}, z_{i}\right)^{T}+\alpha_{j}\left(x_{j}, y_{j}, z_{j}\right)^{T}+\alpha_{k}\left(x_{k}, y_{k}, z_{k}\right)^{T}+\alpha_{l}\left(x_{l}, y_{l}, z_{l}\right)^{T}
$$

To compute $I(\mathbf{W}(\mathbf{x} ; \mathbf{p}))$ in Eq. (5) we do the following: For every point $\mathbf{x}$ in the mean tetrahedrization $\mathbf{s}_{\mathbf{0}}$ compute $\mathbf{W}(\mathbf{x} ; \mathbf{p})$ and sample image $I$ at that location by trilinear interpolation. By a straightforward extension of [6] from 2-D to 3-D,

$$
\frac{\partial \mathbf{W}}{\partial \mathbf{p}}=\sum_{i=1}^{m}\left[\frac{\partial \mathbf{W}}{\partial x_{i}} \frac{\partial x_{i}}{\partial \mathbf{p}}+\frac{\partial \mathbf{W}}{\partial y_{i}} \frac{\partial y_{i}}{\partial \mathbf{p}}+\frac{\partial \mathbf{W}}{\partial z_{i}} \frac{\partial z_{i}}{\partial \mathbf{p}}\right]
$$


where $\frac{\partial \mathbf{W}}{\partial x_{i}}=\left(\alpha_{i}, 0,0\right)^{T} \pi(\mathbf{x}, i), \frac{\partial \mathbf{W}}{\partial y_{i}}=\left(0, \alpha_{i}, 0\right)^{T} \pi(\mathbf{x}, i), \frac{\partial \mathbf{W}}{\partial z_{i}}=\left(0,0, \alpha_{i}\right)^{T} \pi(\mathbf{x}, i)$ and $\frac{\partial x_{i}}{\partial \mathbf{p}}=\left(\mathbf{s}_{1}^{x_{i}}, \mathbf{s}_{2}^{x_{i}}, \ldots, \mathbf{s}_{n}^{x_{i}}\right), \frac{\partial y_{i}}{\partial \mathbf{p}}=\left(\mathbf{s}_{1}^{y_{i}}, \mathbf{s}_{2}^{y_{i}}, \ldots, \mathbf{s}_{n}^{y_{i}}\right), \frac{\partial z_{i}}{\partial \mathbf{p}}=\left(\mathbf{s}_{1}^{z_{i}}, \mathbf{s}_{2}^{z_{i}}, \ldots, \mathbf{s}_{n}^{z_{i}}\right) . \pi(\mathbf{x}, i)$ equals 1 if $\mathbf{x}$ is in a tetrahedron of $\mathbf{s}_{0}$ having landmark $i$ as its vertex, and is 0 otherwise. $\mathbf{s}_{j}^{x_{i}}, \mathbf{s}_{j}^{y_{i}}, \mathbf{s}_{j}^{z_{i}}$ denote the element of $\mathbf{s}_{j}$ corresponding to $x_{i}, y_{i}$ and $z_{i}$ respectively. The summation in (12) is nonzero only for the 4 vertices of the tetrahedron enclosing the point $\mathbf{x}$ where we are evaluating the jacobian.

By the argument in [6], within first order $\mathbf{W}^{-\mathbf{1}}(\mathbf{x} ; \boldsymbol{\Delta} \mathbf{p})=\mathbf{W}(\mathbf{x} ;-\boldsymbol{\Delta} \mathbf{p})$. From (8) we conclude that we need to find a parameter $\mathbf{p}^{\prime}$ such that $\mathbf{W}\left(\mathbf{x} ; \mathbf{p}^{\prime}\right)=$ $\mathbf{W}(\mathbf{x} ; \mathbf{p}) \circ \mathbf{W}^{-\mathbf{1}}(\mathbf{x} ; \boldsymbol{\Delta} \mathbf{p})$. We can approximate this quantity by finding a $\mathbf{p}^{\prime \prime}$ such that $\mathbf{W}\left(\mathbf{x} ; \mathbf{p}^{\prime \prime}\right)=\mathbf{W}(\mathbf{x} ; \mathbf{p}) \circ \mathbf{W}(\mathbf{x} ;-\boldsymbol{\Delta} \mathbf{p})$. The problem here is that piecewise affine warping does not form a group under the operation of composition. In other words the composition of two piecewise affine warps cannot necessarily be described by another piecewise affine warp. We compensate for this by estimating a new position for the landmarks $\mathbf{s}_{0}$ under the composition of the two warps and once we have done this for all landmarks in $\mathbf{s}_{0}$, we estimate $\mathbf{p}^{\prime \prime}$ by finding the closest vector $\mathbf{p}$ in Eq. (1) satisfying the new landmarks and letting $\mathbf{p}^{\prime \prime}=\mathbf{p}$.

We estimate a new position for landmarks $\mathbf{s}_{0}$ by the following method. For every landmark $\mathbf{a}$ in vector $\mathbf{s}_{0}$ we estimate $\mathbf{W}(\mathbf{a} ;-\boldsymbol{\Delta} \mathbf{p})$ by using Eq. (1) with $-\boldsymbol{\Delta} \mathbf{p}$ as parameter. Then, to compute $\mathbf{W}(\mathbf{W}(\mathbf{a} ;-\boldsymbol{\Delta} \mathbf{p}) ; \mathbf{p})$ we use the following heuristic procedure, which gives good results for the $2-\mathrm{D}$ case in [6] and our $3-\mathrm{D}$ case. For each one of the tetrahedra in $\mathbf{s}_{0}$ having $\mathbf{a}$ as a vertex, we estimate the destination of $\mathbf{W}(\mathbf{a} ;-\boldsymbol{\Delta} \mathbf{p})$ under that tetrahedron's affine warp and we define the value of $\mathbf{W}(\mathbf{W}(\mathbf{a} ;-\boldsymbol{\Delta} \mathbf{p}) ; \mathbf{p})$ to be the average value of the destination of $\mathbf{W}(\mathbf{a} ;-\boldsymbol{\Delta} \mathbf{p})$ under the affine warps of those tetrahedra.

\subsection{Fitting Without $\mathrm{X}$ and $\mathrm{Y}$ Axes Rotations}

Let $\mathbf{x}=(x, y, z)^{T}$ and $\mathbf{q}=\left(q_{1}, q_{2}, q_{3}, q_{4}, q_{5}, q_{6}\right)=\left(\frac{a}{c_{1}}, \frac{b}{c_{2}}, \frac{c}{c_{3}}, \frac{t_{x}}{c_{4}}, \frac{t_{y}}{c_{5}}, \frac{t_{z}}{c_{6}}\right)$, where the $c_{i}$ are constants that will be defined later. Assume $\mathbf{N}(\mathbf{x} ; \mathbf{q})$ is a global transform, which does not rotate the shape around the $\mathrm{x}$ and $\mathrm{y}$ axes, defined as follows:

$$
\mathbf{N}(\mathbf{x} ; \mathbf{q})=\left(\begin{array}{ccc}
1+a & -b & 0 \\
b & 1+a & 0 \\
0 & 0 & 1+c
\end{array}\right)\left(\begin{array}{l}
x \\
y \\
z
\end{array}\right)+\left(\begin{array}{c}
t_{x} \\
t_{y} \\
t_{z}
\end{array}\right) .
$$

Notice that $\mathbf{q}=\mathbf{0}$ gives the identity transformation. If we let $a=k \cos (\theta)-1$, $b=k \sin (\theta)$ and $c=s-1$ then

$$
\mathbf{N}(\mathbf{x} ; \mathbf{q})=\left(\begin{array}{ccc}
k \cos (\theta) & -k \sin (\theta) & 0 \\
k \sin (\theta) & k \cos (\theta) & 0 \\
0 & 0 & s
\end{array}\right)\left(\begin{array}{l}
x \\
y \\
z
\end{array}\right)+\left(\begin{array}{l}
t_{x} \\
t_{y} \\
t_{z}
\end{array}\right) .
$$

This performs a rotation by an angle $\theta$ around the z-axis followed by a scaling by $k$ of the x,y coordinates and a scaling by $s$ of the z-coordinates, followed by a translation by $\left(t_{x}, t_{y}, t_{z}\right)^{T}$. If we replace the $s$ above by $k$, then we are performing a three-dimensional similarity transform where we are not rotating around the x,y axes. In other words Eq. (13) above has slightly more expressive power than 
a typical similarity transform since it allows to scale the z-coordinate values by a value different than the scaling of the $\mathrm{x}, \mathrm{y}$ coordinates. Then $\mathbf{N} \circ \mathbf{W}$ performs both the piecewise affine warp of section 4.1 and the global transform $\mathbf{N}$.

Some may argue against scaling along the $\mathrm{z}$ axis independently from the $\mathrm{x}, \mathrm{y}$ axes scalings, since this is not a similarity transform. But from the test cases we performed later on, this does not lead to incorrect model instances, and instead adds more expressive power to our model, making it easier to fit heart models with different $\mathrm{z}$-axes scalings than the ones in our training set.

As noted above, our base mesh is $\mathbf{s}_{0}=\left(x_{1}^{0}, y_{1}^{0}, z_{1}^{0}, \ldots, x_{m}^{0}, y_{m}^{0}, z_{m}^{0}\right)^{T}$. Let $\mathbf{s}_{1}^{*}=$ $c_{1}\left(x_{1}^{0}, y_{1}^{0}, 0, \ldots, x_{m}^{0}, y_{m}^{0}, 0\right)^{T}, \mathbf{s}_{2}^{*}=c_{2}\left(-y_{1}^{0}, x_{1}^{0}, 0, \ldots,-y_{m}^{0}, x_{m}^{0}, 0\right)^{T}, \mathbf{s}_{3}^{*}=c_{3}\left(0,0, z_{1}^{0}\right.$, $\left.\ldots, 0,0, z_{m}^{0}\right)^{T}, \mathbf{s}_{4}^{*}=c_{4}(1,0,0, \ldots, 1,0,0)^{T}, \mathbf{s}_{5}^{*}=c_{5}(0,1,0, \ldots, 0,1,0)^{T}, \mathbf{s}_{6}^{*}=c_{6}(0,0,1$, $\ldots, 0,0,1)^{T}$ where $c_{i}$ is a constant such that $\mathbf{s}_{i}^{*}$ is of unit length. Then

$$
\mathbf{N}\left(\mathbf{s}_{\mathbf{0}} ; \mathbf{q}\right)=\mathbf{s}_{0}+\sum_{i=1}^{6} q_{i} \mathbf{s}_{i}^{*}
$$

where $q_{1}=\frac{a}{c_{1}}, q_{2}=\frac{b}{c_{2}}, q_{3}=\frac{c}{c_{3}}, q_{4}=\frac{t_{x}}{c_{4}}, q_{5}=\frac{t_{y}}{c_{5}}, q_{6}=\frac{t_{z}}{c_{6}}$. If during the shape alignment we aligned the training data such that their center was at point $(0,0,0)$ then $S^{*}=\left\{\mathbf{s}_{1}^{*}, \mathbf{s}_{2}^{*}, \mathbf{s}_{3}^{*}, \mathbf{s}_{4}^{*}, \mathbf{s}_{5}^{*}, \mathbf{s}_{6}^{*}\right\}$ is an orthonormal set.

The set $S=\left\{\mathbf{s}_{1}, \mathbf{s}_{2}, \ldots, \mathbf{s}_{n}\right\}$ from Eq. (1) is the set of eigenvectors of the covariance matrix. For reasons which will become obvious later, we must make sure that every vector in $S^{*}$ is orthogonal to every vector in $S$. Because $S^{*}$ is not a similarity transform, and due to various sources of error, the alignment procedure we performed earlier might not make the two sets fully orthogonal. We therefore have to orthogonalize the two sets $S^{*}$ and $S$. In our test cases we did it as follows. For every vector in $\mathbf{v} \in S$ we take its projection $\mathbf{v}^{\prime}$ onto the space orthogonal to $S^{*}$ by

$$
\mathbf{v}^{\prime}=\mathbf{v}-\sum_{\mathbf{v}^{*} \in S^{*}}\left(\mathbf{v}^{T} \mathbf{v}^{*}\right) \mathbf{v}^{*}
$$

We call this new set $S^{\prime}$. Then, by using an orthogonalization algorithm such as the Gram-Schmidt algorithm we can transform $S^{\prime}$ into $S^{\prime \prime}$, where now $S^{\prime \prime}$ is an orthonormal set. Then every vector in $S^{*}$ is orthogonal to every vector in $S^{\prime \prime}$.

We need to show what is the Jacobian of $\mathbf{N} \circ \mathbf{W}$ which will be used instead of $\frac{\partial \mathbf{W}}{\partial \mathbf{p}}$ in Eq. (5)-(6), and how to update the parameters from Eq. (7). From [6] it is seen that the jacobian of $\mathbf{N} \circ \mathbf{W}$ is $\left(\frac{\partial \mathbf{N} \circ \mathbf{W}}{\partial \mathbf{q}}, \frac{\partial \mathbf{N} \circ \mathbf{W}}{\partial \mathbf{p}}\right)=\left(\frac{\partial \mathbf{N}}{\partial \mathbf{q}}, \frac{\partial \mathbf{W}}{\partial \mathbf{p}}\right)$.

In the same way noted in section 4.1 , to within first order $(\mathbf{N} \circ \mathbf{W})^{-1}$ $(\mathbf{x} ; \boldsymbol{\Delta} \mathbf{q}, \boldsymbol{\Delta} \mathbf{p})=\mathbf{N} \circ \mathbf{W}(\mathbf{x} ;-\boldsymbol{\Delta} \mathbf{q},-\boldsymbol{\Delta} \mathbf{p})$. We use this approximation to define

$$
\mathbf{s}^{\dagger}=\mathbf{N} \circ \mathbf{W}\left((\mathbf{N} \circ \mathbf{W})^{-\mathbf{1}}\left(\mathbf{s}_{\mathbf{0}} ; \Delta \mathbf{q}, \Delta \mathbf{p}\right) ; \mathbf{q}, \mathbf{p}\right)
$$

(the new locations of the landmarks $\mathbf{s}_{0}$ ) by using the method of section 4.1 for composing two warps. Once we have estimated the new landmark positions $\mathbf{s}^{\dagger}$ from Eq. (17), we need to find new values for $\mathbf{p}$ and $\mathbf{q}$ such that $\mathbf{N} \circ \mathbf{W}\left(\mathbf{s}_{\mathbf{0}} ; \mathbf{q}, \mathbf{p}\right)=\mathbf{s}^{\dagger}$. First notice that 


$$
\mathbf{N} \circ \mathbf{W}\left(\mathbf{s}_{\mathbf{0}} ; \mathbf{q}, \mathbf{p}\right)=\mathbf{N}\left(\mathbf{s}_{\mathbf{0}}+\sum_{i=1}^{n} p_{i} \mathbf{s}_{i} ; \mathbf{q}\right)
$$

which can be rewritten as

$$
\mathbf{N}\left(\mathbf{s}_{\mathbf{0}} ; \mathbf{q}\right)+\left(\begin{array}{ccc}
1+a & -b & 0 \\
b & 1+a & 0 \\
0 & 0 & 1+c
\end{array}\right) \sum_{i=1}^{n} p_{i} \mathbf{s}_{i}
$$

where the summations above are taking place over all vectors $\mathbf{s}_{i} \in S^{\prime \prime}$. The matrix multiplication in (19) above with the $3 m$ dimensional vector $\mathbf{s}_{i}$, indicates the result of multiplying every triple of adjacent $\mathrm{x}, \mathrm{y}, \mathrm{z}$ coordinates by the matrix.

By using (19), we see that $\mathbf{N} \circ \mathbf{W}\left(\mathbf{s}_{\mathbf{0}} ; \mathbf{q}, \mathbf{p}\right)=\mathbf{s}^{\dagger}$ can be rewritten as

$$
\begin{gathered}
\mathbf{s}^{\dagger}=\mathbf{s}_{0}+\sum_{i=1}^{6} q_{i} \mathbf{s}_{i}^{*}+ \\
(1+a)\left(\begin{array}{lll}
1 & 0 & 0 \\
0 & 1 & 0 \\
0 & 0 & 0
\end{array}\right) \sum_{i=1}^{n} p_{i} \mathbf{s}_{i}+b\left(\begin{array}{ccc}
0 & -1 & 0 \\
1 & 0 & 0 \\
0 & 0 & 0
\end{array}\right) \sum_{i=1}^{n} p_{i} \mathbf{s}_{i}+(1+c)\left(\begin{array}{lll}
0 & 0 & 0 \\
0 & 0 & 0 \\
0 & 0 & 1
\end{array}\right) \sum_{i=1}^{n} p_{i} \mathbf{s}_{i} .
\end{gathered}
$$

The three terms in Eq. (20) above that are multiplied by $1+\mathrm{a}$, b and $1+\mathrm{c}$, are orthogonal to the vectors in $S^{*}$, since $S^{*}$ and $S^{\prime \prime}$ are orthogonal. This is most difficult to see for the fourth term in (20) that is multiplied by b. This term is orthogonal to the vectors in $S^{*}$ because for each vector in $S^{*}$, if we switch the values of the $\mathrm{x}$ and $\mathrm{y}$ coordinates and change the sign of one of the two coordinates, the resulting vector still belongs to $\operatorname{span}\left(S^{*}\right)$. From $(20)$ we get

$$
q_{i}=\mathbf{s}_{i}^{*} \cdot\left(\mathbf{s}^{\dagger}-\mathbf{s}_{\mathbf{0}}\right)
$$

which we can use to get

$$
p_{i}=\mathbf{s}_{i} \cdot\left(\mathbf{N}^{-1}\left(\mathbf{s}^{\dagger} ; \mathbf{q}\right)-\mathbf{s}_{\mathbf{0}}\right)
$$

where

$$
\mathbf{N}^{-1}\left(\mathbf{s}^{\dagger} ; \mathbf{q}\right)=\left(\begin{array}{ccc}
1+a & -b & 0 \\
b & 1+a & 0 \\
0 & 0 & 1+c
\end{array}\right)^{-1}\left[\mathbf{s}^{\dagger}-\left(\begin{array}{c}
t_{x} \\
t_{y} \\
t_{z}
\end{array}\right)\right]
$$

and we found the new parameters $\mathbf{p}$ and $\mathbf{q}$. Without the orthonormal set $S^{*}$ we introduced, there would be no efficient way of updating the parameters $\mathbf{p}, \mathbf{q}$.

\section{Experimental Results}

We trained and fitted the model on a data set of 11 short axis cardiac MR image sequences with a temporal resolution of 20 frames. Each image's resolution was $256 \times 256$ pixels. For each frame, the number of slices intersecting the left ventricle 
ranged between 6 and 10. All test cases were done on an Intel Xeon 3.06Ghz with 3GB RAM using MATLAB 7.01.

A manual tracing of the left ventricular endocardium and epicardium was acquired from each image where both the left ventricle's endocardium and epicardium was visible. The endocardial contours were drawn behind the papillary muscles and trabeculae.

We trained two models, one using the data from the 10 frames closest to end-diastole and the other model using the remaining 10 frames closest to endsystole, giving us a total of 110 training samples for each of the two models. We used a leave-one-out approach for each model's training: If we wanted to fit an AAM on a certain patient's MR sequence, we trained our two models on the training examples not belonging to that patient, and on each frame we fit the appropriate model. The fitting process stopped if the error change was below a certain threshold, or if the fitting error had stopped decreasing monotonically.

We compared the accuracy of the algorithm described in this paper and of a standard Gauss-Newton optimizer which simultaneously optimized all the model parameters. We compared our algorithm with a Gauss-Newton optimizer for two reasons: Firstly to emphasize the gain in speed that our algorithm can provide compared to brute force approaches. Secondly to investigate our algorithms fitting accuracy against an exhaustively tested algorithm which is known from previous research [6] to outperform the faster (but less reliable) constant matrix approaches described in the introduction.

We manually translated, rotated and scaled $\mathbf{s}_{\mathbf{0}}$ until our model fitted well the median MR slice of the left ventricle, and then ran the algorithms. Once the segmentation was complete, for each of the image slices intersected by our model, we extracted the endocardial and epicardial contours of the model's intersection with the image. A total of 1473 images were segmented by both algorithms. For each one of the landmarks in the extracted contours we estimated the minimum distance to the curve corresponding to the ground truth. The inverse compositional algorithm gave a mean left ventricle endocardial and epicardial error of $1.6 \pm 1.58 \mathrm{~mm}$ and $1.9 \pm 1.7 \mathrm{~mm}$ and a mean fitting speed of 12.1

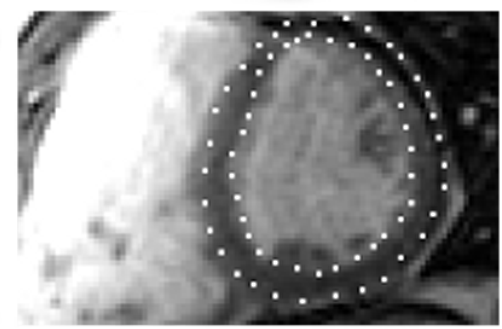

(a)

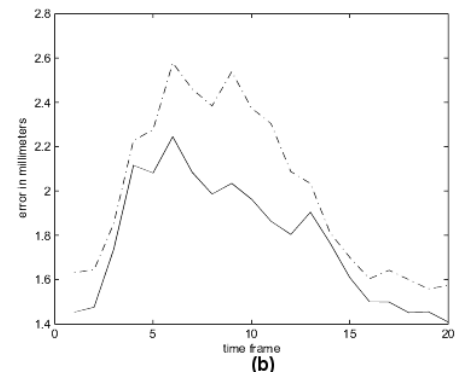

(b)

Fig. 2. (a)Resulting segmentation of an image, shown as white dots. (b) The average error in millimeters for each of the 20 frames in our MR sequences. The solid line is the inverse compositional algorithm's error and the dashed line is the Gauss-Newton error. End-systole is close to frame 10 for the patients in our data set 
seconds. The respective measures for Gauss Newton based optimization were $1.73 \pm 1.61 \mathrm{~mm}$ and $2.20 \pm 2.04 \mathrm{~mm}$ and 688.5 seconds. In Figure 2(b) we show the mean error rates as a function of time frame. The algorithm described in this paper fits the model approximately 60 times faster than a typical brute force Gauss-Newton optimization with a significantly smaller error than standard Gauss-Newton optimization, especially for frames closest to end-systole. We have also experimented with training sets of other sizes, which does lead to significant changes in the error measures we get, but in all cases the inverse compositional algorithm is at least comparable, if not better than Gauss-Newton. We suspect that a reason why Gauss-Newton gives a slightly greater error is because Gauss-Newton has to optimize the appearance parameters also, while the inverse compositional algorithm projects them out. We believe that if both algorithms directly optimized the same number of parameters, Gauss-Newton would be at least as accurate as the inverse compositional algorithm, but would remain much slower. Our models were made up of 30 shape parameters, 20 appearance parameters (that the inverse compositional algorithm projected out) and the 6 global shape parameters $\mathbf{q}$, for a total of 56 parameters. The GaussNewton based models used 5 global shape parameters (the z-axis scaling was the same as that of the x,y axes) so they used 55 parameters. The inverse compositional algorithm proved to be extremely sensitive to global intensity changes in the images. We handled this by normalizing the left ventricle's intensity in each image to a common mean and standard deviation before training and fitting our models.

\section{Conclusions}

We presented an efficient and robust algorithm for fitting 3-D AAMs on short axis cardiac MRI. It gives rapid segmentation results with a precision that is at least as good as that of previously reported results and brute force GaussNewton optimization. In conclusion, the fitting algorithm presented here has given us encouraging results, indicating that it might become the method of choice for fitting 3-D AAMs when rotation around only one axis is needed.

\section{Acknowledgements}

We thank Dr. Paul Babyn and Dr. Shi-Joon Yoo of the Department of Diagnostic Imaging at the Hospital for Sick Children in Toronto for providing us the MRI data. JKT holds a Canada Research Chair in Computational Vision and acknowledges its financial support. AA holds an NSERC PGS-M and acknowledges its financial support. We also thank the reviewers for their comments. 


\section{References}

1. S. Baker, R. Goss, and I. Matthews, "Lucas-Kanade 20 Years on: A Unifying Framework," International Journal of Computer Vision, Vol. 56, No. 3, pp. 221-255, 2004.

2. S. Baker, and I. Matthews, "Equivalence and Efficiency of Image Alignment Algorithms," In Proceedings of the IEEE Conference on Computer Vision and Pattern Recognition, Vol. 1, pp. 1090-1097, 2001.

3. T.F. Cootes, "Statistical Models of Appearance for Computer Vision," [Online]. Available: http://www.isbe.man.ac.uk/ bim/Models/app_models.pdf

4. T.F. Cootes, G. Edwards, and C. Taylor, "Active Appearance Models," In Proceedings of the European Conference on Computer Vision, Vol. 2, pp. 484-498. 1998.

5. A.F. Frangi, W.J. Niessen, and M.A. Viergever, "Three-Dimensional Modeling for Functional Analysis of Cardiac Images: A Review," IEEE Transactions on Medical Imaging, Vol.20, No. 1, pp. 2-25, 2001.

6. I. Matthews, and S. Baker, "Active Appearance Models Revisited," International Journal of Computer Vision, Vol. 60, No. 2, pp. 135-164, 2004.

7. S. C. Mitchell, J. G. Bosch, B. P. F. Lelieveldt, R. J. van der Geest, J. H. C. Reiber, and M. Sonka, "3-D Active Appearance Models: Segmentation of Cardiac MR and Ultrasound Images," IEEE Transaction on Medical Imaging, Vol.21, No. 9, pp. 1167-1178, 2002. 